\title{
An Underactuated Wearable Arm-swing Rehabilitator for Gait Training
}

\author{
Owen R. Barnes, Babak Hejrati, and Jake J. Abbott
}

\begin{abstract}
This paper presents the design concept and fabricated prototype of a device that swings the arms for use in gait rehabilitation. The device is designed to be used in conjunction with a body-weight-support treadmill. The device is backdrivable, wearable, capable of assisting the user's arm swing in the sagittal plane, and has unhindered kinematics in the remaining unactuated degrees of freedom. Tests are performed to validate the shoulder-angle prediction equations based on the non-collocated motor-angle sensor measurements, to validate the device's ability to provide adequate torque to induce arm-swing in a passive user, and to investigate whether or not the user's active involvement can be determined by examining sensor data. The results show that the device does provide sufficient torque to move the arms with a factor of safety, but that the model-based shoulder-angle estimates obtained from the motor measurements have non-negligible error with the current prototype. It is shown that the controlled device generates low RMS tracking error and is able to diagnose user-assistance level (i.e., if the user is passive or actively assisting arm swing) online by observing shoulder-angle amplitudes and peak motor torques.
\end{abstract}

\section{INTRODUCTION}

The walking gait of those who have had strokes or spinalcord injury (SCI) is often altered so that it is no longer healthy, but these people can undergo physical therapy in order to improve gait. Rehabilitation is done through exercises that help stimulate muscles and exploit neuroplasticity for the diminished functions [1]. Gait rehabilitation is often focused on the legs and de-emphasizes the role of arms. However, studies show that there is neural coupling between the upper and lower limbs [2] and that it can be exploited for rehabilitative purposes [3]. Research also shows that upperlimb muscle activity can actually induce lower-limb muscle activity [4], [5] and that the effect is most pronounced when the arms move in phase with the legs [6]. Additionally, arm swing contributes to balance [2], regulates rotational body motion [7], and metabolic efficiency of the walker [8]. Therefore, more effective rehabilitation can be performed as the patient exerts effort to naturally swing their arms.

One method of gait rehabilitation involving arm swing was shown in a study in which SCI subjects walked on a treadmill with their arms being manually assisted by a therapist with poles [2]. This type of rehabilitation enabled the subject to exercise both the upper and lower limbs. However, according to [9], rehabilitation is activity-dependent, and using devices (especially ones with arm supports) can alter the input interpreted by the spinal cord, thus leading to

This material is based upon work supported by the National Science Foundation under Grant No. 1208637.

The authors are with the Department of Mechanical Engineering, University of Utah, USA. \{owen.barnes, babak.hejrati, jake.abbott\} eutah. edu

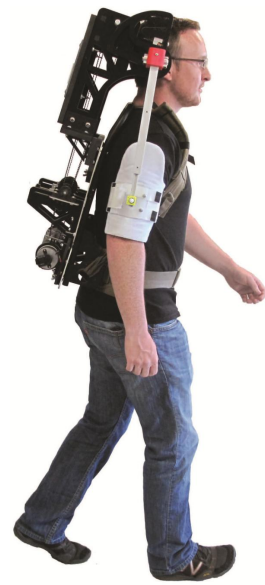

(a)

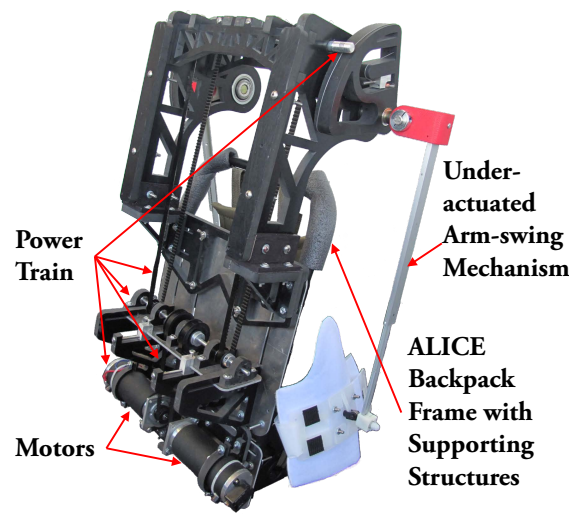

(b)
Fig. 1. (a) The UWEAR is worn like a backpack, and provides active arm-swing assistance for flexion/extension of the shoulder, while being unconstraining in the other degrees of freedom. (b) The UWEAR comprises several subassemblies: a backpack frame with additional supporting structures, an underactuated arm-swing mechanism, and a power train that transmits motor torque to torque for the arm-swing mechanism.

the learning of incorrect muscle firing patterns. Although the arm weight that is supported by the therapist's poles may be little, depending on the therapist's skill, it may be enough to cause the learning of incorrect muscle firing patterns. Therefore, it is important to allow the arms to swing as naturally as possible without gripping or supporting weight. Additionally, this method of rehabilitation requires several physical therapists to assist the patient during the exercise.

Many robotic technologies have been developed for performing gait rehabilitation [10]-[14], but the vast majority are focused on the legs with no active assistance for arm swing. One example of a robotic orthosis includes arm-swing assistance [13]. The robot consists of swinging prismatic links with handholds that interact with the user's hands and arms, combined with sliding height- and pitch-adjustable foot pads. Since the robotic system constrains the user's feet and arms kinematically, it is likely that what the user experiences is dissimilar to natural, over-ground walking.

The need for a device that properly swings the arms during gait training for neurorehabilitative purposes has led to the development of the Underactuated WEarable Armswing Rehabilitator (UWEAR), shown in Fig. 1. The device is powered in just one degree of freedom (DOF) to assist in flexion/extension of the user's shoulder, while allowing relatively uninhibited motion of the user's arms in the remaining DOFs. The UWEAR is worn like a backpack on the user while they are walking on a treadmill. Body-weight- 
support is already provided for the user, which can also be used to compensate the additional weight of the UWEAR. Its arm links move in flexion/extension and abduction/adduction. The range of motion is large $\left(-40^{\circ}\right.$ extension, $90^{\circ}$ flextion, and $20^{\circ}$ abduction), and covers the motions necessary for both natural gait and relatively free movement while not performing rehabilitative tasks. Our goal was not to design a fully powered portable exoskeleton, but rather a therapeutic device that assists the patient's arms in following a healthy gait at their own walking pace. The UWEAR comprises three key subassemblies: a military All-purpose Lightweight Individual Carrying Equipment (ALICE) frame with additional supporting structures, underactuated arm-swing mechanisms to induce arm swing in the shoulder joint, and a power train to convert torque generated by DC motors located near the user's hips to amplified torque near the user's shoulders for the arm-swing mechanisms.

The underactuated arm-swing mechanism applies power to the user's arms in the sagittal plane without constraining the arms in the other unactuated DOFs. The assemblies are located lateral to the user's arms. They start above the user's shoulders, near the user's head, from the UWEAR's supporting structures, and extend to the user's arms via arm cuffs. The assemblies comprise five joints each, all with one DOF. Only the shoulder flexion/extension DOF is actuated. The underactuated arm-swing mechanism was designed, and is described here, independently of the power train that powers its single actuated DOF.

A military ALICE backpack frame provides both a foundation for the rest of the mechanism and a secure fit on the user. The ALICE frame is made of aluminum and steel. The strength and rigidity of the metals along with the adjustable shoulder and waist straps accomplish two objectives. They provide adequate reaction forces to ensure that power is spent in moving the arms, rather than moving the frame relative to the user's body. Additionally, the strength and rigidity of the frame prevent the structure from flexing from the torques generated by the motors.

Additional structural components support the underactuated arm-swing mechanism and power train. ABS is chosen for its strength and weight. Screws fasten two slotted aluminum plates to the ALICE frame. The slots enable positioning the device's components and enable modular additions (e.g., the power train's tensioning shelf and motor mounts). Several bolts and slots in the structure provide adjustability for the UWEAR so that it fits a large population.

The power train-comprising motors, a timing-belt system, and capstan drives-is located on the back of the ALICE frame. The timing-belt system transfers torque from the motors, which sit by the user's hips, up to the input of the underactuated arm-swing mechanism, located above the user's shoulders. The timing-belt assembly has stages of pulleys that amplify the motor torques. After the first stage of pulleys there is a tensioning device, and by adjusting its positioning screws, it can eliminate slack in the timing-belts. Large motors with no gearhead provide relatively high torque while being backdrivable. The power train's final stage is the capstan drive, which further amplifies the torque while maintaining the backdrivability of the power train.

\section{DESIGN OF THE UWEAR}

\section{A. Underactuated Arm-swing Mechanism}

Fig. 2(a) shows the underactuated arm-swing mechanism comprising a 2-DOF shoulder joint, a 1-DOF sliding prismatic link, and a 2-DOF cuff joint. The shoulder joint is made of two custom 1-DOF joints. They enable powered flexion/extension and free abduction/adduction.

The prismatic arm link is a 1-DOF sliding joint. Because there is an offset between the user's shoulders and the mechanism's shoulder joint, as well as movement that can occur from the user's scapulothoracic joint, as well as to accommodate users of varying size, an arm link made of sliding rails is used to account for necessary change in link length as the user flexes/extends and abducts/adducts their arm. Otherwise, the user would experience constrained kinematics. Telescopic slide rails from MISUMI (\#SAR230) are used for the prismatic arm links; they cover the necessary range of lengths encountered in flexion/extension and abduction/adduction in normal walking.

The cuff joint is made of three components: a small bearing housing, a pin joint formed by an eyelet and clevis rod end, and an arm cuff. The small bearing housing accommodates rotational differences between the user's upper arm and the mechanism's arm link in flexion/extension. The eyelet and clevis rod end pin joint accommodate angular differences between the user's upper arm and the mechanism's arm link in abduction/adduction. The arm cuff has sheet plastic attached to it that passes through the clevis rod end. This prevents the rod end from rotating about an axis normal to the arm cuff's surface, which prevents the clevis rod end's abduction/adduction axis from changing orientations that would cause awkward and uncontrollable pulling motions. The arm cuff is worn firmly on the user's upper arm so that forces generated by the UWEAR are transmitted to the user.

\section{B. Power Train and Supporting Structures}

The power train is made of motors, a timing-belt system, and a capstan drive. Its purpose is to amplify and transmit motor torque to the arm-swing mechanisms. The DC motors (Brush Type DC Servo Motor from Servo Systems \#23SMDC-LCSS-500) are direct-drive and backdrivable. The motors are sufficiently short such that they do not obstruct the user's arms as the arms swing past the motor's location. The motors have a maximum continuous stall torque of $0.388 \mathrm{~N} \cdot \mathrm{m}$, which is sufficient for generating arm swing when combined with the additional torque amplification of the drive train. The backdrivability of the motors and drive train make the UWEAR unconstraining when it is unpowered, which is desirable for fail-safe operation and easy donning/doffing of the device. The motors are placed near the user's hips with the goal of mitigating additional rotational inertia on the user.

The timing-belt system comprises two stages of timingpulleys and timing-belts that span the distance between the 


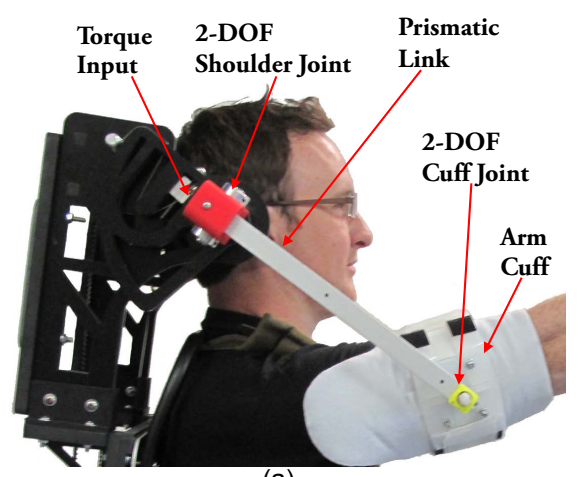

(a)

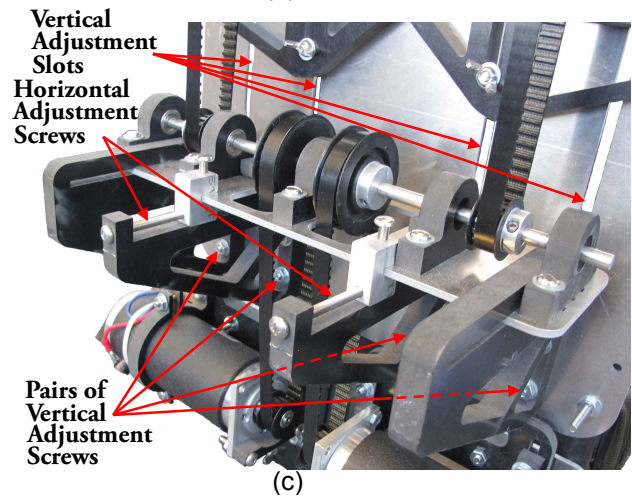

(c)

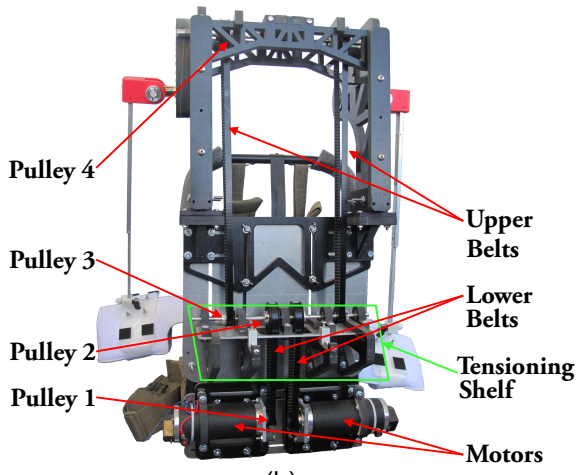

(b)

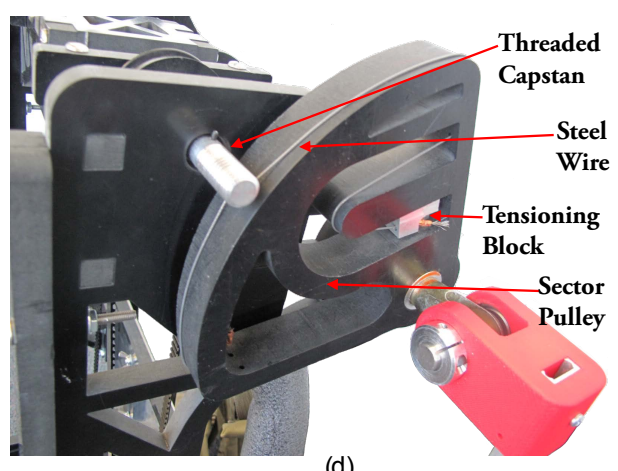

(d)

Fig. 2. Several images of the UWEAR prototype. (a) highlights the underactuated arm-swing mechanism, (b) highlights the timing-belt system, (c) focuses on the timing-belt system's tensioning shelf, and (d) highlights the capstan drive.

motor shaft and input shaft of the capstan drive (Fig. 2(b)). The timing-pulleys are of two different pitch diameters, $46.89 \mathrm{~mm}$ and $22.63 \mathrm{~mm}$, which result in a total timing-belt system gear ratio of $k_{T B}=4.30$. The timing-belts span the stages of pulleys, and have a belt-width of $9.53 \mathrm{~mm}$, which prevents belt skipping from potential timing-belt teeth deflections. The timing-belt system includes an adjustable device for tensioning the belts called the "tensioning shelf" (Fig. 2(c)), which ensures good torque transmission as well as facilitates the timing-belt system's assembly.

The last member of the power train is the capstan drive (Fig. 2(d)). The capstan drive draws inspiration from various "haptic paddle" designs [15], [16]. It provides one final stage of torque amplification. It is made of a threaded capstan, which transmits torques via a steel wire (diameter $=0.94 \mathrm{~mm}$ ) that rotates the sector pulley, which is the input to the armswing mechanism. The capstan drive assembly also includes a tensioning block to eliminate slack in the steel wire. The threaded capstan has a radius of $6.35 \mathrm{~mm}$, a length of $25.4 \mathrm{~mm}$, and thread count of 13 threads-per-inch such that the steel wire does not unravel from the capstan during operation (from overrunning either the length of the capstan or the wire over the individual threads from poor steel-wire diameter sizing). The sector pulley is designed to be large in radius $(12.29 \mathrm{~cm})$ so that a large gear ratio for the capstan drive is obtained $\left(k_{C D}=19.36\right)$. The gear ratio for the entire power train is the product of the timing-belt system's and capstan drive's gear ratios; it is $k_{P T}=83.2$.

The ALICE frame and its straps serve the important purpose of providing a foundation to mount the rest of the UWEAR components and providing a stable connection between the UWEAR and user, so that minimal relative motion between them occurs. The rigidity of the ALICE frame as well as the lateral supports and truss bridge insure that the generated torques are applied to the user's arm, rather than causing the device to deflect.

The total weight of the UWEAR is about $10 \mathrm{~kg}$, however, a standard body-weight-support system can compensate the total weight of the device. A rehabilitation harness can be worn underneath the ALICE frame, such that the UWEAR to be worn simultaneously with a body-weight-support system, as depicted in Fig. 3, which shows the UWEAR being worn by a mannequin combined with a standard body-weightsupport system. In this way, the weight of the UWEAR can be compensated along with the weight of the user.

\section{GEOMETRY OF THE ARM-SWING MECHANISM}

The arm-swing mechanism can be described geometrically in order to create a relationship between the user's shoulder angle and the mechanism's arm-link angle. Fig. 4 presents the geometry used, in two different configurations: when the upper arm is vertical (the "zero" position) and when the upper arm is flexed to an arbitrary shoulder angle $\theta_{s}$. Parameters $O_{s}$ and $O_{m}$ represent the user's shoulder axis and the arm-swing mechanism's powered axis, respectively. The distance between $O_{s}$ and $O_{m}$ is described by $D$. The angle between the line measured by $D$ and vertical is described by 


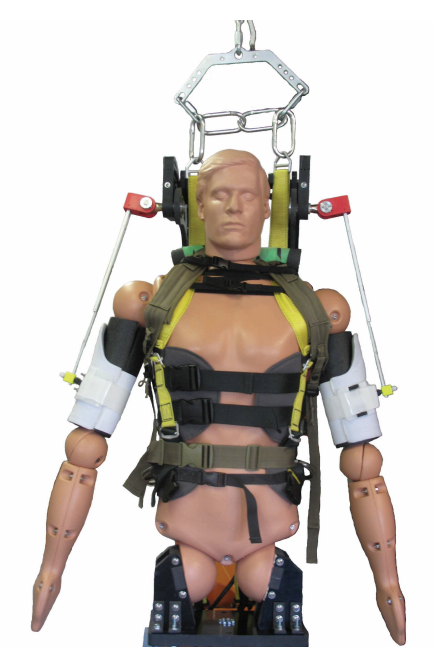

Fig. 3. The UWEAR was designed to be worn in conjunction with a weight-support system.

$\gamma$. The relative angle of the prismatic arm link is represented by $\theta_{m} . O_{c}$ is the connection point between the mechanism and the user's upper arm at an arbitrary shoulder angle. $O_{c 0}$ is the connection point's location at the "zero" position. $R$ represents the distance between the user's shoulder axis and the connection point. The length of the mechanism's prismatic link, $L\left(\theta_{s}\right)$, is a function of $\theta_{s}$. At the "zero" position $\left(\theta_{s}=0^{\circ}\right)$, the initial length of the prismatic arm link is represented by $L_{0}$. The angle $\alpha$ describes the angle between the user's upper arm and the prismatic link in the "zero" position and $\phi$ represents the angle between $D$ and $L_{0}$. A number of additional useful relationships follow:

$$
\begin{gathered}
\alpha=\arctan \left(\frac{D \sin \gamma}{D \cos \gamma+R}\right) \\
\phi=\gamma-\alpha \\
L_{0}=\frac{R \sin (\pi-\gamma)}{\sin \phi}=\frac{R \sin (\gamma)}{\sin \phi}
\end{gathered}
$$

It is now possible to find the relationship between the shoulder angle and the mechanism angle. First, the length of the prismatic link is calculated as:

$$
L=D \cos \left(\theta_{m}-\phi\right)+\sqrt{R^{2}-D^{2} \sin ^{2}\left(\theta_{m}-\phi\right)}
$$

The Law of Cosines can then used, first on the triangle $O_{m} O_{c 0} O_{c}$ and then on the triangle $O_{s} O_{c 0} O_{c}$, which share the side $\rho$, to find the cosine of the user's shoulder angle:

$$
\cos \left(\theta_{s}\right)=1-\frac{L^{2}+L_{0}^{2}-2 L L_{0} \cos \left(\theta_{m}\right)}{2 R^{2}}
$$

However, use of the arccos function to solve for $\theta_{s}$ can be poorly conditioned numerically. Equation (5) is rearranged to a more numerically robust form using a trigonometric halfangle formula involving the tangent and cosine of the same angle:

$$
\theta_{s}= \pm 2 \arctan \sqrt{\frac{1-\cos \left(\theta_{s}\right)}{1+\cos \left(\theta_{s}\right)}}
$$

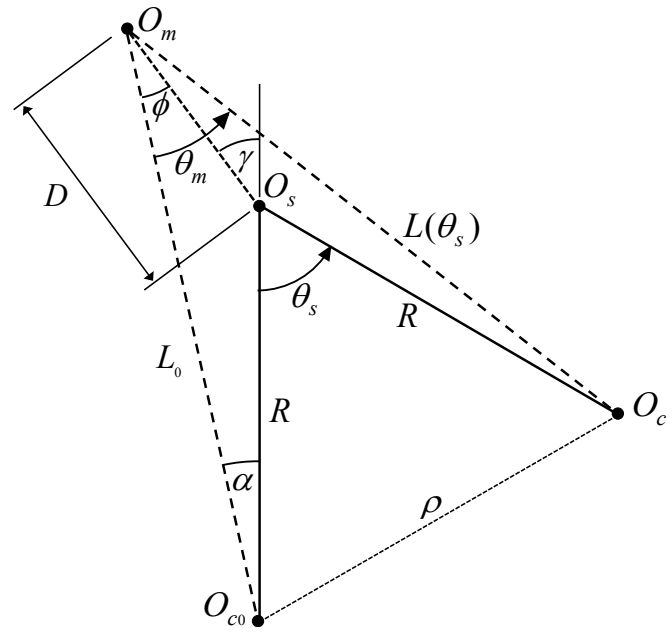

Fig. 4. Geometry of the powered DOF of the arm-swing mechanism, shown in two different configurations: with the upper arm vertical, which we refer to as the "zero" position, and with the upper arm flexed to an arbitrary shoulder angle. The parameters that are used for calculating the relationship between the arm-swing mechanism and the user's shoulder angle are shown.

Substituting the solutions for $\cos \left(\theta_{s}\right)$ from (5) into (6) gives the final relationship to calculate the user's shoulder angle based on the measured angle of the arm-swing mechanism:

$$
\theta_{s}= \pm 2 \arctan \sqrt{\frac{L^{2}+L_{0}^{2}-2 L L_{0} \cos \left(\theta_{m}\right)}{4 R^{2}-L^{2}-L_{0}^{2}+2 L L_{0} \cos \left(\theta_{m}\right)}}
$$

The positive solution for $\theta_{s}$ is used when $\theta_{m}$ is positive, the negative solution is used when $\theta_{m}$ is negative, and $\theta_{s}$ is zero when $\theta_{m}$ is zero

The geometric model here assumes that the shoulder joint is a static pin joint. However, the shoulder joint is capable of moving due to its scapulothoracic degrees of freedom. Therefore, (7) is not a relationship that will predict the shoulder angle with high accuracy, but rather it will approximate it. This result is seen in the experiments of Section IV.

\section{EXPERIMENTAL RESULTS}

In the experiments, the UWEAR is worn by four healthy male subjects with heights $\{1.71,1.77,1.71,1.91\}$ in meters and masses of $\{80,65,70,94\}$ in kilograms. Only four subjects were used here because we are only interested in validating the performance of the UWEAR prototype, not in conducting any human-subjects study per se.

After the UWEAR is donned and has its straps tightened so that it is secure, measurements are made to obtain values for $R, D, L_{0}$, and $\gamma$, which are used to estimate the user's shoulder angle from the mechanism's angle.

\section{A. Validation of the Relationship between the Sector Pulley and Shoulder Angle}

An experiment was performed to evaluate the accuracy of the geometrical relationship provided in (7), which uses motor encoder data combined with the total power-train gear ratio to estimate the user's shoulder angle, compared against 
angles obtained by using motion-capture cameras to accurately measure the relative angle between the user's upper arm and torso without any assumptions about the shoulder's kinematics. One test subject donned the UWEAR and was fitted with motion-capture markers in standard locations. The subject, after starting from a relaxed position with his arms at his side, moved his arms periodically between the rangeof-motion limits (approximately from $-40^{\circ}$ extended to $90^{\circ}$ flexed) for a trial time of 60 seconds. The absolute errors between the motion-capture and encoder-based trajectories are shown in Fig. 5(a). It is seen that the error of the shoulder-angle prediction equations are not larger than $12^{\circ}$, with maximum errors that occur at a position outside the normal range of arm-swing motion $\left(-30^{\circ}\right.$ extension to $10^{\circ}$ flexion [8]). Additionally, errors appear to decrease as armmotion speeds increase toward those of natural arm swing. The error is non-negligible, and it is believed that this is largely due to the subject's shoulder movement (Fig. 5(b)), which is also non-negligible, since (7) assumes that the user's shoulder is an immovable pin joint. Thus, we conclude that the UWEAR, in its current form, cannot be used for highaccuracy position measurement.

\section{B. Inducing Arm-swing}

1) Experiment Design: Another experiment is performed to characterize the UWEAR's ability to induce arm-swing in its users under a variety of different factors including arm-swing frequency $(0.6 \mathrm{~Hz}$ or $1.0 \mathrm{~Hz}$, which correspond to a slow or a brisk walking pace, respectively [17]), and user assistance level (passive, in which the user relaxes their arms, and assistive, in which the user attempts to swing their arms as being directed by the UWEAR, using only haptic information). The desired sinusoidal shoulder-angle trajectory for inducing arm-swing is precalculated based on the limited information in [18]. A position tracking PD servo controller with gains of $k_{p}=2.0 \mathrm{~N} \cdot \mathrm{m} / \mathrm{rad}$ and $k_{d}=$ $0.3 \mathrm{~N} \cdot \mathrm{m} \cdot \mathrm{s} / \mathrm{rad}$ is implemented in the UWEAR to track the desired trajectory. The gains are tuned to be stiff yet stable to minimize tracking error.

Each of the four subjects stand with their arms initially at their sides. The UWEAR is then activated and it swings their arms through $20^{\circ}$-amplitude sinusoidal motion $\left(-30^{\circ}\right.$ extension to $10^{\circ}$ flexion) while motor-torque and opticalencoder data is recorded. To test all the factors and levels, the subjects perform 4 trials each with randomized order. The trials are evaluated by examining the peak motor torques, RMS tracking error, and shoulder-angle amplitudes once the transient from the beginning of the trial has decayed (after 5 seconds).

2) Results and Discussion: Fig. 6 contains the data for the experiment. Fig. 6(a) shows the peak motor torques required by the UWEAR for different frequencies and assistance levels. The required peak motor torque for any case is not higher than $0.12 \mathrm{~N} \cdot \mathrm{m}$, which is approximately one-third of the continuous stall torque that the chosen motor can provide. Thus, we see that the selected motors are oversized, and that

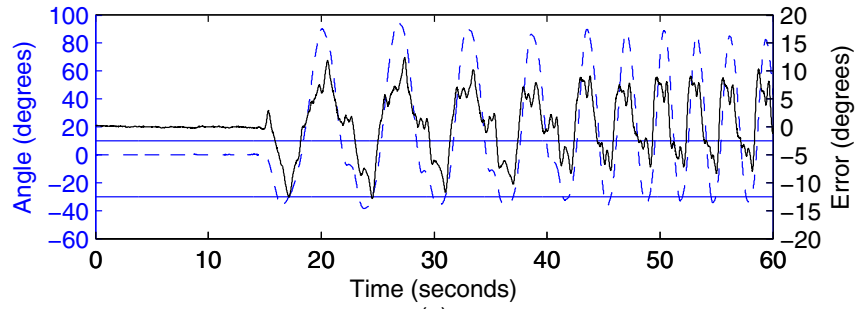

(a)

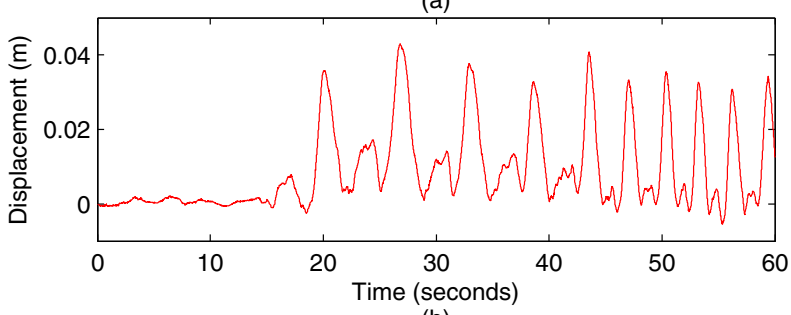

(b)

Fig. 5. (a) Errors between the motion-capture and encoder-based data for the shoulder-angle, in black (right vertical axis), compared against the motion-capture data for the shoulder angle, in dashed blue (left vertical axis). Blue horizontal reference lines at $10^{\circ}$ and $-30^{\circ}$ show the expected range of arm swing during normal gait. (b) Vertical displacement of the shoulder joint, obtained from the motion-capture data.

they could be chosen to be less powerful, with the potential benefit of being more lightweight.

Fig. 6(b) shows the shoulder-angle amplitudes created by the UWEAR for different frequencies and assistance levels. With increasing frequency, the shoulder-angle amplitude increases, and the assistive user case creates shoulder-angle amplitudes larger than the passive case. At $1.0 \mathrm{~Hz}$, it is seen that the assistive user case has a median shoulder-angle amplitude larger than the desired of $20^{\circ}$.

The RMS tracking errors of the UWEAR are shown in Fig. 6(c). The errors increase with increasing arm-swing frequency, but there appears to not be a difference between RMS error for the user assistance level. The RMS errors are not larger than approximately $1.6^{\circ}$.

The UWEAR can diagnose the level of user assistance by examining the peak motor torque and shoulder-angle amplitudes. When examining the motor torques, significant differences exist between the user assistance levels for motor torque at both frequencies. At $0.6 \mathrm{~Hz}$, the assistive level requires less motor torque than the passive; however, at $1.0 \mathrm{~Hz}$, the assistive level requires more motor torque. This may be due to the user's errors in following the desired trajectory, which requires more torque, since the PD controller is error based. The user assistance level can also be diagnosed by observing the shoulder-angle amplitudes at both tested armswing frequencies. For both frequencies, the assistive user case achieves significantly greater shoulder-angle amplitudes than the passive user case. As discussed previously, the errors for predicting the shoulder angles are non-negligible, but they do not prevent the shoulder-angle amplitudes from being used to monitor user involvement for rehabilitation for the same therapy session, since the movement of the shoulder joint appears fairly repeatable for a given user during a given session. The RMS errors have no significant 


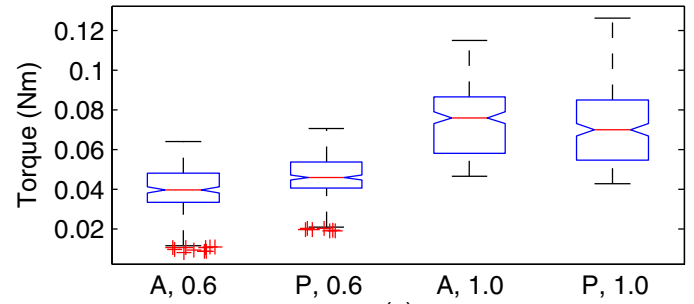

(a)

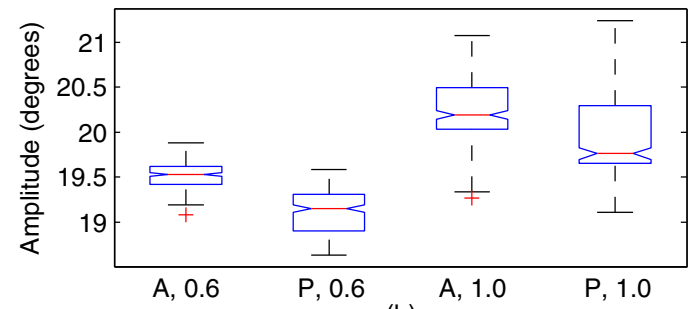

(b)

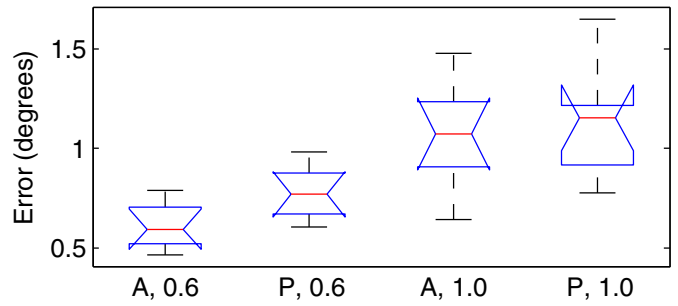

User Assistance Level, and Arm-Swing Frequency

(c)

Fig. 6. Box plots showing the results of the human-subject experiments. The subfigures contain the data for (a) the maximum motor torques, (b) shoulder-angle amplitude, and (c) RMS error. The individual boxes are coded by the user assistance level $(\mathrm{A}=$ assistive, $\mathrm{P}=$ passive $)$, and arm-swing frequency $(1.0=1.0 \mathrm{~Hz}, 0.6=0.6 \mathrm{~Hz})$. Note that the desired shoulder-angle amplitude is $20^{\circ}$. In a box plot, the red line in the center indicates the median of the data. The upper and lower blue edges that bound the box indicate the 75 th and 25 th percentile of the data, respectively. The dashed black lines above and below the boxes-the whiskers-extend to the most extreme data points that are not considered outliers. Outliers are plotted as red crosses, if they are present. The notches centered around the medians of the box plots indicate the $95 \%$ confidence interval for the median, and indicate whether the median is significantly different from that of another box, depending on if the boxes' notches overlap or not.

differences between user assistance levels and cannot be used to diagnose user involvement.

\section{CONCLUSIONS}

The UWEAR has promise of being a successful device for inducing arm-swing. It is a therapeutic device designed to be used along with a body-weight-support during gait rehabilitation on a treadmill. Its design makes it free of kinematic constraints for the user's arms. The error associated with the geometric relationship between the sector pulley and user's shoulder angle (due to unmodeled shoulder-joint movements) is non-negligible at the lower and upper limits of the UWEAR's motion range; the device is not to be used for high accuracy positioning. The UWEAR induces arm swing in its users and can diagnose the user assistance level via motor torque and shoulder-angle amplitudes. A remaining open problem is how to generate proper armswing trajectories, to be tracked by UWEAR, in real-time based on the user's self-determined walking.

\section{ACKNOWLEDGMENT}

The authors would like to thank Dr. John Hollerbach and Dr. Andrew Merryweather for their helpful comments.

\section{REFERENCES}

[1] A. L. Behrman, M. G. Bowden, and P. M. Nair, "Neuroplasticity after spinal cord injury and training: an emerging paradigm shift in rehabilitation and walking recovery,' Physical Therapy, vol. 86, no. 10, pp. 1406-1425, 2006.

[2] A. L. Behrman and S. J. Harkema, "Locomotor training after human spinal cord injury: A series of case studies," Physical Therapy, vol. 80, no. 7, pp. 688-700, 2000.

[3] D. S. Marigold and J. E. Misiaszek, "Whole body responses: Neural control and implications for rehabilitation and fall prevention," The Neuroscientist, vol. 15, no. 1, pp. 36-46, 2009.

[4] D. P. Ferris, H. J. Huang, and P.-C. Kao, "Moving the arms to activate the legs," Exercise and sport science reviews, vol. 34, no. 3, pp. 113 120, 2006.

[5] F. Sylos-Labini, Y. P. Ivanenko, M. J. MacLellan, G. Cappellini, R. E. Poppele, and F. Lacquanti, "Locomotor-like leg movements evoked by rhythmic arm movements in humans," PLOS ONE, vol. 9, no. 3, 2014.

[6] D. de Kam, J. Duysens, and V. Dietz, "Do we need allowing arm movements for rehabilitation of gait?" in Converging Clinical and Engineering Research on Neurorehabilitation, J. L. Pons, D. Torricelli, and M. Pajaro, Eds. Springer-Verlag, 2013, pp. 959-962.

[7] H. Elftman, "The function of the arms in walking," Human Biology, vol. 11 , no. 4, pp. 529-535, 1939.

[8] S. H. Collins, P. G. Adamczyk, and A. D. Kuo, "Dynamic arm swinging in human walking." Proceedings. Biological sciences / The Royal Society, vol. 276, no. 1673, pp. 3679-3688, 2009.

[9] N. J. Tester, D. R. Howland, K. V. Day, S. P. Suter, A. Cantrell, and A. L. Behrman, "Device use, locomotor training, and the presence of arm swing during treadmill walking post-spinal cord injury," Spinal Cord, vol. 49, no. 3, pp. 451-456, 2011.

[10] R. Riener, L. Lunenburger, S. Jezernik, M. Anderschitz, G. Colombo, and V. Dietz, "Patient-cooperative strategies for robot-aided treadmill training: First experimental results," IEEE Trans. Neural Systems and Rehabilitation Engineering, vol. 13, no. 3, pp. 380-394, 2005.

[11] J. F. Veneman, R. Kruidhof, E. E. G. Hekman, R. Ekkelenkamp, E. H. F. Van Asseldonk, and H. van der Kooij, "Design and evaluation of the LOPES exoskeleton robot for interactive gait rehabilitation," IEEE Trans. Neural Systems and Rehabilitation Engineering, vol. 15, no. 3, pp. 379-386, 2007.

[12] Y. Stauffer, Y. Allemand, M. Bouri, J. Fournier, R. Clavel, P. Metrailler, R. Brodard, and F. Reynard, "The WalkTrainer-a new generation of walking reeducation device combining orthoses and muscle stimulation," IEEE Trans. Neural Systems and Rehabilitation Engineering, vol. 17, no. 1, pp. 38-45, 2009.

[13] J. Yoon, B. Novandy, C. H. Yoon, and K. J. Park, "A 6-dof gait rehabilitation robot with upper and lower limb connections that allows walking velocity updates on various terrains," IEEE Trans. Mechatronics, vol. 15, pp. 201-215, 2010.

[14] H. Schmidt, S. Hesse, C. Werner, and A. Bardeleben, "Upper and lower extremity robotic devices to promote motor recovery after stroke- recent developments," in Proc. Int. Conf. IEEE EMBS, 2004, pp. $4825-4828$.

[15] A. M. Okamura, C. Richard, and M. R. Cutkosky, "Feeling is believing: Using a force-feedback joystick to teach dynamic systems," J. Engineering Education, vol. 91, no. 3, pp. 345-349, 2002.

[16] K. Bowen and M. O'Malley, "Adaptation of haptic interfaces for a LabVIEW-based system dynamics course," in 14th Symposium on Haptic Interfaces for Virtual Environment and Teleoperator Systems, 2006, pp. 147-152.

[17] H. van Hedel, L. Tomatis, and R. Muller, "Modulation of leg muscle activity and gait kinematics by walking speed and bodyweight unloading," Gait and Posture, vol. 24, pp. 35-45, 2006.

[18] J. Perry, Gait Analysis: Normal and Pathological Function. SLACK Incorporated, 1992. 